\title{
Editorial
}

\section{Nutrition Education for the Health Care Professions}

\author{
Martin Kohlmeier, ${ }^{1,2}$ Caryl A. Nowson, ${ }^{3}$ Rose Ann DiMaria-Ghalili, ${ }^{4}$ and Sumantra Ray ${ }^{5,6}$ \\ ${ }^{1}$ Department of Nutrition, School of Medicine and Gillings School of Global Public Health, University of North Carolina at \\ Chapel Hill, 800 Eastowne Drive, Suite 100, Chapel Hill, NC 27514, USA \\ ${ }^{2}$ UNC Nutrition Research Institute, 500 Laureate Way, Kannapolis, NC 28081, USA \\ ${ }^{3}$ Centre for Physical Activity and Nutrition Research, School of Exercise and Nutrition Sciences, Deakin University, \\ Waurn Ponds Campus, Locked Bag 20000, Geelong, VIC 3220, Australia \\ ${ }^{4}$ College of Nursing and Health Professions, Drexel University, Philadelphia, PA, USA \\ ${ }^{5}$ UK Need for Nutrition Education/Innovation Programme in Partnership with MRC Human Nutrition Research in Cambridge and \\ the British Dietetic Association, c/o Elsie Widdowson Laboratory, Cambridge CB1 9NL, UK \\ ${ }^{6}$ Cambridge University Hospitals and School of Clinical Medicine, c/o Elsie Widdowson Laboratory, Cambridge CB1 9NL, UK
}

Correspondence should be addressed to Martin Kohlmeier; mkohlmeier@unc.edu

Received 22 June 2015; Accepted 22 June 2015

Copyright (C) 2015 Martin Kohlmeier et al. This is an open access article distributed under the Creative Commons Attribution License, which permits unrestricted use, distribution, and reproduction in any medium, provided the original work is properly cited.

Nutrition and related lifestyle factors greatly impact wellbeing in health and disease. The World Health Organization defines nutrition as "the intake of food, considered in relation to the body's dietary needs. Good nutrition-an adequate, well balanced diet combined with regular physical activityis a cornerstone of good health. Poor nutrition can lead to reduced immunity, increased susceptibility to disease, impaired physical and mental development, and reduced productivity" (http://www.who.int/topics/nutrition/en/). At the level of the population, we are grappling with the challenges of the double burden of both over- and undernutrition. Despite the profound impact good nutrition has on health and wellness, the science of nutrition and its application to health care are not fully integrated in most health professions training programs. This gap is further compounded by the fact that patients and the public remain confused about the correct nutritional advice to follow given the widespread media interest attracted by diet and the disparity in nutritionrelated health messages that are in circulation. While dietitians are recognized as the health care professionals with nutrition expertise, all health care professionals need to be knowledgeable and competent in nutrition as it applies to health promotion and prevention, as well as treating acute and chronic diseases. Fewer than 100,000 registered dietitians and other state-licensed nutrition professionals practice in the USA. This modest number is eclipsed by the more than 3 million physicians, physician assistants, nurses, pharmacists, dentists, and many other groups of US health care professionals. The distribution is similar in many other countries. For example, in Australia there are 2,831 dietitians: 0.03 per 1,000 compared to the ratio of physicians ( 3 per 1,000). In the UK, the number of dietitians to doctors in the health service is 3 per 100. Even in the presence of robust referral mechanisms between doctors and dietitians there still needs to be effective nutritional screening and triage by doctors in order to provide specialist input to the most appropriate patients. As a result, most of the non-nutritionist health care providers have to deal with nutrition-related conditions and diseases on a daily basis, but few are adequately prepared to recognize key diagnostic signs and then assist their patients and clients with effective interventions. This need for adequate nutritional training has global relevance because there is no region or country without significant impact of nutrition on health outcomes. Health care providers in developing countries often have to worry about different nutrition problems in their patients, but their need for adequate nutrition training is just as great as that of their colleagues in more affluent regions and is also often unmet. Recent exchanges between a number of countries have highlighted the fact that there is much in common with the nature of the problems relating to gaps 
in medical and health care nutrition education worldwide. Potential solutions may also rely on common denominators.

The evidence base for practical use of nutrition-based preventive and curative interventions is rapidly growing and implementation research steadily improves delivery of best practices. There is little doubt that health professionals can be more effective in their daily practice when they draw on current nutrition knowledge and effective clinical skills. The challenge is to fit a core set of this nutrition information, based on synthesis of enduring evidence, into current undergraduate and postgraduate health care training schedules that are already bursting at the seams. In response to this challenge, the current issue focuses on nutrition education for the health care professions. The articles in this issue focus on 4 themes: current status of nutrition content in osteopathic and medical schools in the USA, innovative programs for integration of nutrition content in medical and osteopathic schools in the USA and Australia, knowledge gaps of practicing health care professionals in Australia, the UK and more widely, and translating nutrition research from the bench to the bedside in the USA and Australia.

An important question is how much nutrition education for future health care providers is enough. Thirty years ago, a panel commissioned by the Institute of Medicine in the USA mandated a minimum of 25-30 content hours of nutrition for medical school curricula [1]. In a recent survey of all US medical schools, K. M. Adams et al. found that most medical schools are not meeting this recommendation. Even more alarming is that the first ever survey conducted by K. B. Early et al. on nutrition content in osteopathic schools in the USA also follows a similar trend. This is concerning since osteopathic training by its nature is more holistic with the assumption that nutrition content would be more fully integrated in osteopathic schools than the traditional medical schools.

Even though most medical and osteopathic schools fall short of the required 25 hours of content, medical and osteopathic school faculty continue to implement innovative educational programs to integrate nutrition into existing curriculum. L. A. Hark et al. provide examples of how readily available resources can be integrated into medical schools to meet the LCME standards. A. Chatterjee et al., S. E. Ettinger et al., and J. King et al. provide examples of how to involve medical students in university or community-based nutrition programs to gain valuable experience by integrating nutrition knowledge in practice.

N. Schoendorfer and J. Schafer, R. Connor et al., J. Crowley et al., and W. E. Hardman et al. discuss how nutrition is integrated in their respective medical school curriculum in the USA and Australia. L. Scalfi et al. explain the structures of curricula for nutritionists in Italy. A common finding among these papers is that medical students recognize the importance of nutrition in chronic disease prevention and also acknowledge the need for more nutrition content in their programs early on.

A logical conclusion is that if health professionals do not receive adequate nutrition content during their training programs, this would lead to a knowledge gap when in practice. C. A. Nowson and S. L. O'Connell discuss the nutrition knowledge gap of Australian general practitioners and P. Douglas et al. discuss the knowledge and practice gaps related to dietitian-led hydration management in the UK. This also reflects the fact that some areas of nutritional management, such as hydration, are overlooked more than others. Ray et al. explore nutrition knowledge in a snapshot of doctors and dietitians in a region of India and whilst there are similarities with Western paradigms, there are also key differences. These papers clearly demonstrate the need for continued education in nutrition-related topics for all health care professionals.

The last set of papers in this special issue focus on translating nutrition research into practice across the health care professions. K. C. Wohlgenant et al. describe an innovative program delivered by nurses on food safety prevention in older adults in the USA. Z. E. Davidson and C. Palermo highlight the development of a research competency for undergraduate nutrition students in Australia. S. E. Ettinger et al. discuss a unique postgraduate fellowship program to prepare the nonphysician nutrition scientist for clinical nutrition research-moving discoveries from bench to bedside.

Reading across the piece, on one hand there is a need to position the nutrition education of health care professions such that it is informed by current research and high quality evidence whilst on the other hand continuing research into the clinical effectiveness of new educational initiatives. This is not a new concept but simply reflects the "Knowledge to Action" approach that connects research, education, and practice in many other disciplines. It is time that we consider its application to nutrition.

What our joint efforts underscore is the need for a forum where investigators and practitioners can share insights on the state of nutrition education for health care professionals and innovative instructional practices for improving their nutrition competencies.

We hope these articles will inspire nutrition educators and instructional investigators across the globe to further develop new programs or replicate existing ones, to enable nutrition into the curriculum of health professions education at the undergraduate and graduate levels.

\section{Martin Kohlmeier \\ Caryl A. Nowson \\ Rose Ann DiMaria-Ghalili \\ Sumantra Ray}

\section{References}

[1] Committee on Nutrition in Medical Education, Food and Nutrition Board, Commission on Life Sciences, and National Research Council, Nutrition Education in U.S. Medical Schools, The National Academies Press, Washington, DC, USA, 1985. 

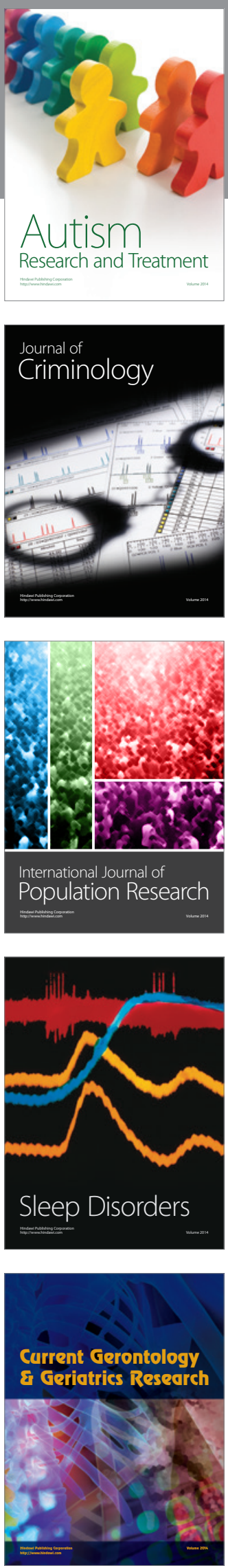
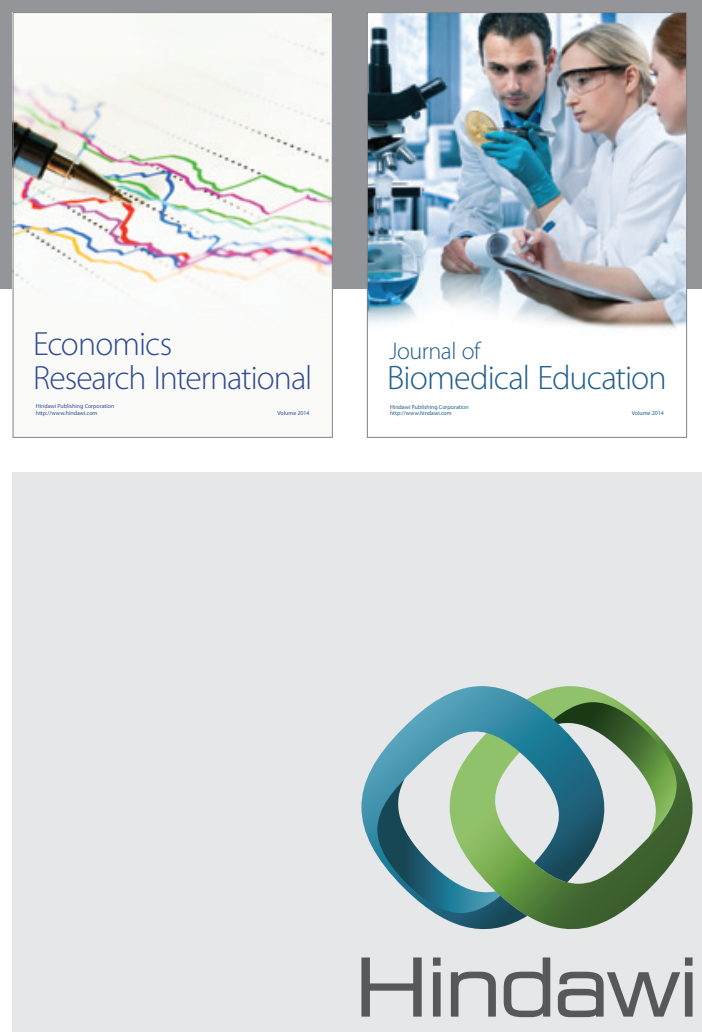

Submit your manuscripts at

http://www.hindawi.com
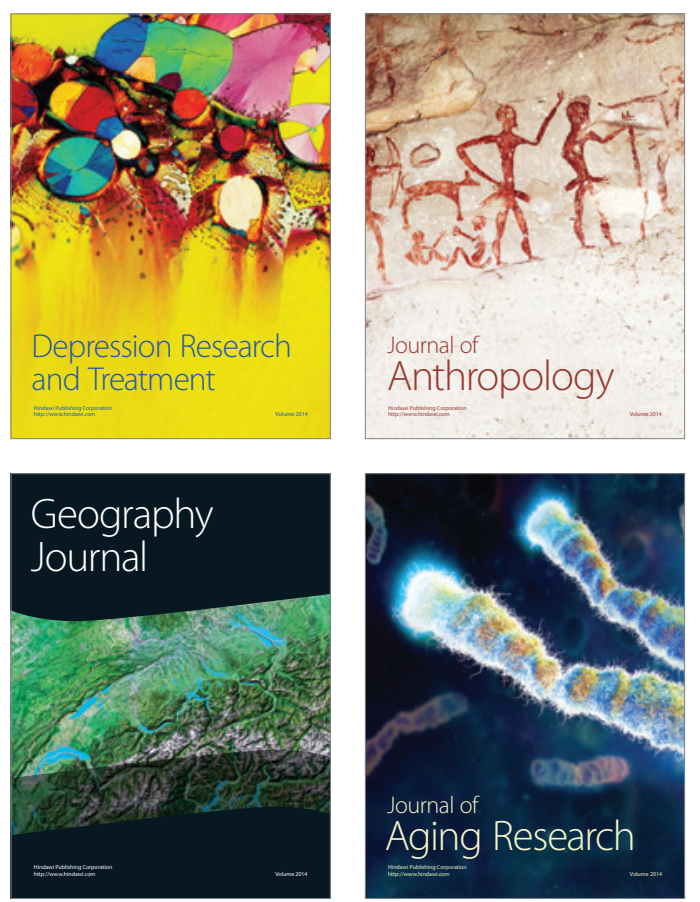
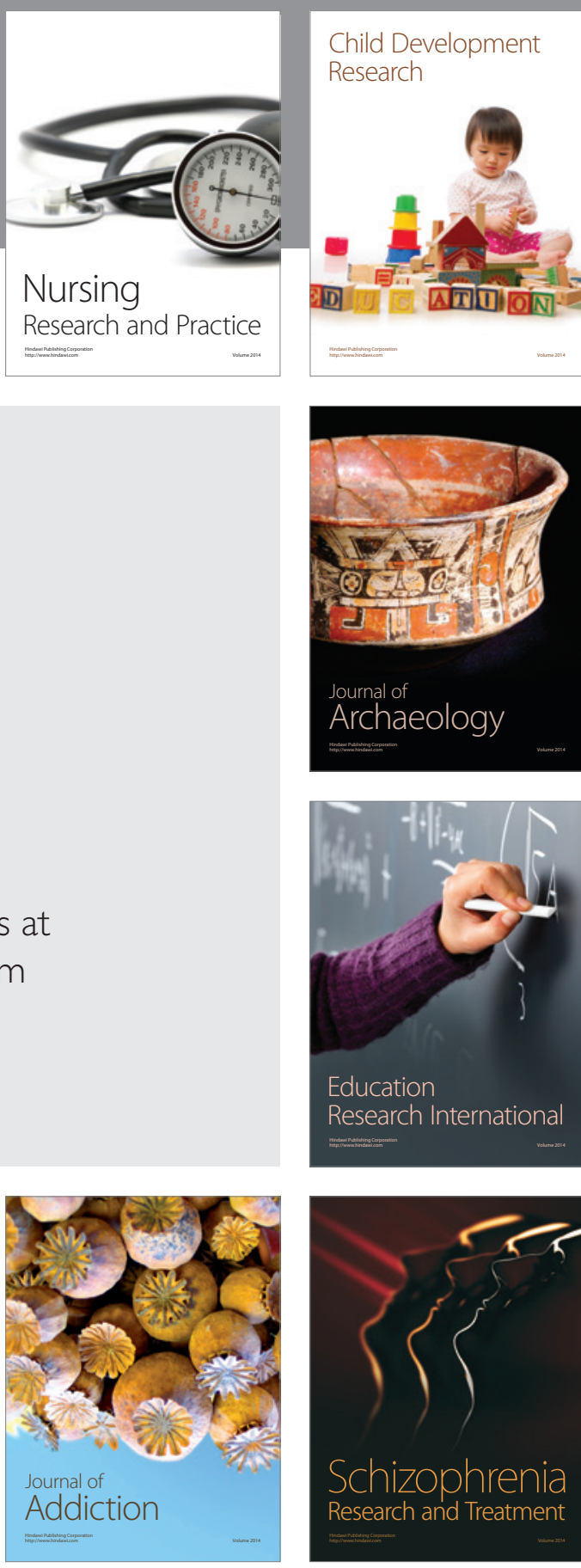

(D)
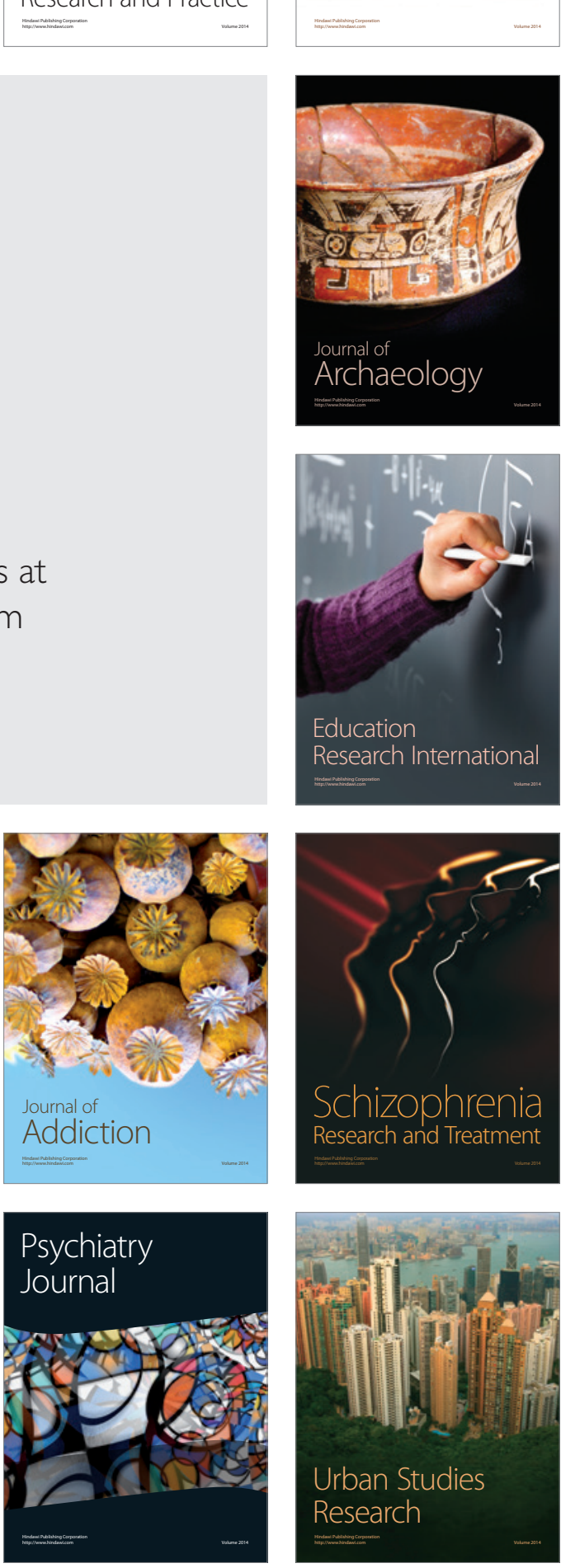